\title{
A Compact Gamma Camera for Biological Imaging
}

\author{
Eric L. Bradley, Julie Cella, Stan Majewski, Vladimir Popov, Jianguo Qian, Margaret S. Saha, Member, IEEE, \\ Mark F. Smith, Member, IEEE, Andrew G. Weisenberger, and Robert E. Welsh
}

\begin{abstract}
A compact detector, sized particularly for imaging a mouse, is described. The active area of the detector is approximately $46 \mathrm{~mm} \times 96 \mathrm{~mm}$. Two flat-panel Hamamatsu $\mathrm{H8500}$ position-sensitive photomultiplier tubes (PSPMTs) are coupled to a pixellated $\mathrm{NaI}(\mathrm{Tl})$ scintillator which views the animal through a copper-beryllium $(\mathrm{CuBe})$ parallel-hole collimator specially designed for ${ }^{125} \mathrm{I}$. Although the PSPMTs have insensitive areas at their edges and there is a physical gap, corrections for scintillation light collection at the junction between the two tubes results in a uniform response across the entire rectangular area of the detector. The system described has been developed to optimize both sensitivity and resolution for in-vivo imaging of small animals injected with iodinated compounds. We demonstrate an in-vivo application of this detector, particularly to SPECT, by imaging mice injected with approximately $10-15 \mu \mathrm{Ci}$ of ${ }^{125} \mathrm{I}$.
\end{abstract}

Index Terms-Biomedical imaging, scintillation detectors, single photon emission computed tomography, small animal imaging.

\section{INTRODUCTION}

I $\mathrm{N}$ order to perform effective in vivo imaging of small animals or specific organs, it is desirable that the detector produce a seamless image of the entire animal or organ at sufficiently high resolution and sensitivity. Moreover, the detector should be compact, economical, and readily allow future expansion of the system. As reviewed in several recent reports, there has been significant progress in achieving these goals using a variety of approaches and detector systems [1]-[8]. Recently, a new position sensitive photomultiplier tube (PSPMT), the H8500, has become generally available [9]. Several research groups have tested the new tube [10]-[19] and several of these investigators have reported on its suitability for nuclear medicine imaging applications. Pani and collaborators [10], [11] have tested this device for both uniformity and spatial resolution and have com-

Manuscript received November 29, 2004; revised November 5, 2005. This work was supported in part by the U.S. National Institute of Health under Grant EB000458, in part by the Office of Biological and Environmental Research of the Office of Science of the U. S. Department of Energy, in part by the Department of Defense Breast Cancer Research Program, in part by the U.S. National Science Foundation, in part by the Thomas F. and Kate Miller Jeffress Memorial Trust, and in part by the HHMI Undergraduate Biological Sciences Education Program Grant to The College of William and Mary. The Southeastern Universities Research Association operates Thomas Jefferson National Accelerator Facility for the U. S. Department of Energy under Contract DE-AC05-84ER40150.

E. L. Bradley and M. S. Saha are with the Department of Biology, The College of William and Mary, Williamsburg, VA 23187 USA (e-mail: elbrad@wm.edu).

J. Cella and R. E. Welsh are with the Department of Physics, The College of William and Mary, Williamsburg, VA 23187 USA.

S. Majewski, V. Popov, M. F. Smith, and A. G. Weisenberger are with the Thomas Jefferson National Accelerator Facility, Newport News, VA 23606 USA.

J. Qian is with the Department of Applied Science,The College of William and Mary, Williamsburg, VA 23187 USA.

Digital Object Identifier 10.1109/TNS.2005.862977 pared their results using both $\mathrm{CsI}(\mathrm{Tl})$ and $\mathrm{NaI}(\mathrm{Tl})$ pixellated scintillators in a range of pixel sizes. The technique reported by Popov et al. [16] has been applied in the work described here so as to yield good detector sensitivity across the $3 \mathrm{~mm}$ gap between the two tubes' photocathodes at the junction between the glass envelopes of the PSPMTs. Rouze et al. [18] and Lerche et $a l$. [14] have demonstrated the suitability of this PSPMT when applied to PET imaging of small animals. Stolin et al. [17] have compared the H8500 to the larger R3292 PSPMT and have applied the technique of Popov et al. [16] to an assembly of twelve such tubes. Techniques of charge division among the H8500's pad anodes have been studied by Olcott et al. [15]. Applicability of the H8500 to detection of neutrons and high-energy gamma rays has been studied by Engels et al. [12] while Korpar and collaborators [13] have employed this tube in a ring imaging Cerenkov detector.

Building upon the results reported with the H8500 PSPMT described in these studies, we have sought to devise a very compact detector capable of imaging an entire mouse, and also one which will facilitate the simultaneous use of several such detectors arranged symmetrically in close proximity to the animal. Our principal goal is the construction of a gamma camera compact enough so that eventually four or more identical cameras can surround a mouse for use in dynamic SPECT imaging. By contrast, a symmetric array of four RS3292 PSPMTs with larger width (diameter) would necessarily be positioned farther from the subject animal with consequent poorer spatial resolution.

We have incorporated two such tubes into a small, compact gamma-ray camera of a size particularly suitable for imaging mice. In this design, a pair of these tubes has been combined and attached to a pixellated $\mathrm{NaI}(\mathrm{Tl})$ crystal array [20] with a $\mathrm{CuBe}$ parallel-hole collimator designed for use with compounds tagged with ${ }^{125} \mathrm{I}$. The detector package is enclosed in an aluminum box suitable for mounting on a rotating gantry for use both in planar imaging and SPECT. Lead foil was inserted in the $\mathrm{Al}$ box to shield the detector from radiation not arriving via the collimator. The collimator parameters have been selected to provide suitable system resolution (slightly over $2 \mathrm{~mm}$, full-width half-maximum (FWHM) on contact with the collimator), and good sensitivity for imaging mice injected with only $10 \mu \mathrm{Ci}$ of ${ }^{125} \mathrm{I}$ and placed about $20 \mathrm{~mm}$ from the detector face. As more than 400 compounds of biological interest are available commercially tagged with ${ }^{125} \mathrm{I}$, this isotope can be applied in a range of imaging studies. The relatively long halflife (60 days) permits the acquisition and storage of a quantity of the tagged material without ready access to production facilities. The fact that shorter-lived iodine isotopes are used in a number of human applications provides useful comparison with related studies in small animals as discussed here. The relatively low photon en- 


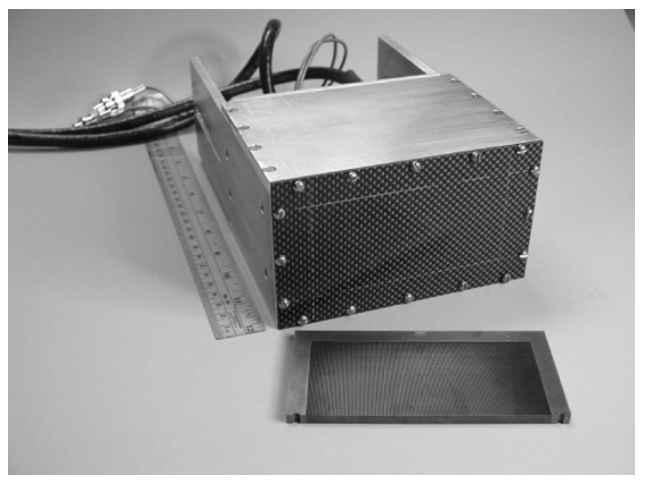

Fig. 1. Completed detector with an identical collimator shown lying in front of the detector.

ergy emitted by ${ }^{125}$ I does, however, place some demands on detector design and performance as discussed below.

Shown in Fig. 1 is the assembled gamma camera and a collimator identical to the one used in these studies. Here we report the design and construction of this system as well as a brief description of a "proof of principle" series of imaging studies of the uptake of sodium-iodide in a lactating female mouse. The studies carried out with this detector indicate that this combination of PSPMT, scintillator/pixel size and collimator construction provides a well-matched gamma imager for studies of the distribution of ${ }^{125} \mathrm{I}$ in a mouse. Measurements to study the suitability of this detector to both planar imaging and SPECT applications are described and the particular utility of an array of four or more such compact detectors for dynamic in vivo SPECT with ${ }^{125} \mathrm{I}$ is discussed.

\section{Design of the Gamma Camera}

We have reported previously on a number of imaging devices based on the $120 \mathrm{~mm}$ diameter, round Hamamatsu R3292 PSPMT [22]-[27]. We have carried out those studies employing both parallel-hole and pinhole collimators with a range of spatial resolutions. When applying our single- or dual-head imaging devices in a rotating gantry with the goal of achieving useful SPECT images, we have noted some limitations. We have found that SPECT reconstruction can readily be achieved when the subject is a euthanized mouse. In such cases where there is no metabolism, a relatively long time can be taken to acquire a series of planar images with a high signal to noise ratio with as little as $10 \mu \mathrm{Ci}$ of activity. For a live mouse, we have found that this same level of injected activity allows the acquisition of acceptable planar images even while the tagged material is being metabolized throughout the animal's body. Owing to the relatively rapid distribution of most such ligands throughout the living mouse body, the goal of reliable SPECT reconstruction imposes the demand for high activity and relatively brief planar imaging. An obvious solution to this limitation is the use of several identical detectors placed symmetrically in a compact array around the body of the mouse. We have therefore designed and constructed a detector that is considerably more compact than one based on the R3292 PSPMT. The new "mouse-sized" gamma camera described here utilizes a pair of the new H8500 PSPMTs. A compact array of four or more identical gamma cameras can provide a symmetrical system to surround a mouse and permit acquisition of planar images simultaneously from several angles potentially permitting imaging in a short period relative to the changing distribution of the ligand throughout the body of the living mouse.

\section{CONSTRUCTION OF THE IMAGER}

\section{A. Hamamatsu H8500 PSPMT}

The H8500 is a square, flat-panel PSPMT with external dimensions of $52 \mathrm{~mm} \times 52 \mathrm{~mm} \times 34 \mathrm{~mm}$ thick and a sensitive area approximately $49 \mathrm{~mm} \times 49 \mathrm{~mm}$. In order to image an entire mouse, gamma cameras based on more than one such tube will generally be required. It is a 12 -stage tube with a gain of $10^{6}$ and an $8 \times 8$ array of anode pads. This device has recently become generally available. Standard specimens of that tube are sold with anode output uniformity not exceeding $5: 1$. The selected tubes used in this device exhibited sensitivity variations of about 2:1.

\section{B. NaI(Tl) Pixellated Scintillation Crystal}

The scintillator employed in this detector is of pixellated $\mathrm{NaI}(\mathrm{Tl})$ and is manufactured by Bicron-St. Gobain [20]. The crystal array has an active area of about $98 \mathrm{~mm} \times 48 \mathrm{~mm}$ to match the active region of the pair of adjoined phototubes. The pixel dimensions are $1 \mathrm{~mm} \times 1 \mathrm{~mm} \times 5 \mathrm{~mm}$ with a pitch of $1.2 \mathrm{~mm}$. The array is viewed through a $3 \mathrm{~mm}$ glass window and encapsulation is completed by an aluminum cover $50 \mu \mathrm{m}$ thick. The $\mathrm{Al}$ entrance window results in approximately $15 \%$ attenuation of photons in the $30 \mathrm{keV}$ energy range applicable to studies with ${ }^{125}$ I. Owing to the difficulty in cutting and sealing arrays of such small pixels of $\mathrm{NaI}(\mathrm{Tl})$, the manufacturer made a protective outer perimeter of $2 \mathrm{~mm}$ pixels for added integrity of the array. The chosen pixel depth $(5 \mathrm{~mm})$, while greater than necessary for efficient absorption of the $\sim 30 \mathrm{keV}$ photons from ${ }^{125} \mathrm{I}$, permits application of the camera to higher energy photons and provides sufficient light collection at the PSPMT as evidenced by good signal to noise and clear image definition.

\section{Parallel-Hole Collimator}

For initial studies of this system, a $\mathrm{CuBe}$ parallel-hole collimator has been employed. The collimator, fabricated by Tecomet, Inc. [21], is $5 \mathrm{~mm}$ thick and has an active area $52 \mathrm{~mm}$ $\times 105 \mathrm{~mm}$. The collimator, shown in Fig. 1, measures $58 \mathrm{~mm}$ $\times 122 \mathrm{~mm}$ in area owing to the presence of a frame on three sides of the active rectangular area. Two such collimators were produced by cutting a square $122 \mathrm{~mm}$ on each edge through the central axis. It has square cells $0.55 \mathrm{~mm}$ on each side separated by $0.11 \mathrm{~mm}$ thick septum walls. Collimator dimensions were based on the formulae given by Smith et al. [28] as discussed further in Section IV below.

\section{Assembly of the Detector}

A box measuring $140 \mathrm{~mm} \times 82 \mathrm{~mm} \times 107 \mathrm{~mm}$ deep machined of $8 \mathrm{~mm}$ thick $\mathrm{Al}$ is used to contain the detector system. The front face entrance window is of $2.0 \mathrm{~mm}$ thick graphite-based composite material manufactured principally for use in medical imaging tables [29]. The presence of that window resulted in an $8 \%$ reduction of transmission of photons 
emitted by ${ }^{125} \mathrm{I}$. Mounting brackets extend from two sides for positioning in a frame or gantry. Inside the Al box, the two H8500 PSPMTs are gently attached to each other with flexible cement along an edge and then taped against the glass face of the $\mathrm{NaI}(\mathrm{Tl})$ scintillator array. An optical grease [30] is used for optical coupling of the PSPMTs to the scintillation array window. The $\mathrm{CuBe}$ collimator is held in place inside the box against the front entranced window by brackets along the short sides. Changing the collimator can be effected by removing the screws which hold the composite entrance window to the Al box. The combination of the two PSPMTs and the NaI(Tl) array are gently pressed against the back of the collimator within the box.

\section{E. Anode Readout}

Although each of the PSPMTs has 64 anode pads in an 8 by 8 array, the signals are read out from each tube via a matrix of 128 resistors (one " $\mathrm{X}$ " and one "Y" per pad) arranged to allow the $\mathrm{X}$ and the $\mathrm{Y}$ signals from one tube to be represented by 8 signal lines for each dimension [16]. Sixteen amplified outputs from each PSPMT are connected to a 16-channel FERA LeCroy CAMAC ADC (two ADCs are used). The Kmax-based [31] data acquisition algorithm calculates the digitized signals from both PSPMTs to permit a single continuous active detector surface in one of the detection coordinates. As the two PSPMTs do not have identical gain at the same bias voltage, they are operated at different high voltages and this is currently achieved with a single HV power supply and a resistive divider.

\section{RESULTS: EFFICIENCY AND RESOLUTION}

A raw crystal image of the detector (left panel in Fig. 2) shows the presence of the transition region at the gap between the two phototubes, however all the scintillation pixels are seen and are well-separated. This image was made using $662 \mathrm{keV}$ gamma rays from a ${ }^{137} \mathrm{Cs}$ source positioned $150 \mathrm{~mm}$ from the front face of detector with the $\mathrm{CuBe}$ collimator in place. The individual pixels are readily identified at this higher energy range. The raw crystal array image is then imported into a special Kmax program for identification of the geometric response region of each crystal and the development of a crystal pixel lookup table ( $36 \times 80$ pixels) followed by energy calibration of each crystal element pixel using a source of ${ }^{125} \mathrm{I}$. A large liquid planar flood source containing $40 \mu \mathrm{Ci}$ of ${ }^{125} \mathrm{I}$ placed above the $\mathrm{CuBe}$ collimator permitted the formation a flood-correction table to obtain the relative efficiency of each pixel. As is evident in the center image in Fig. 2, the gap between the individual PSPMTs does not prevent reliable identification of each crystal element. No detection efficiency loss or visible deterioration of performance was observed in the gap region between the PSPMTs. Uniformity of the detector's efficiency is further exhibited in the image of a source comprised of three glass capillaries of inside diameter $0.3 \mathrm{~mm}$ and length about $120 \mathrm{~mm}$. Each capillary was loaded with approximately $10 \mu \mathrm{Ci}$ of ${ }^{125} \mathrm{I}$ (right image in Fig. 2). A profile image through those capillaries is shown in Fig. 3. We note that the images of the radioactive capillaries in the rightmost panel of Fig. 2 do not show discontinuities at the interface

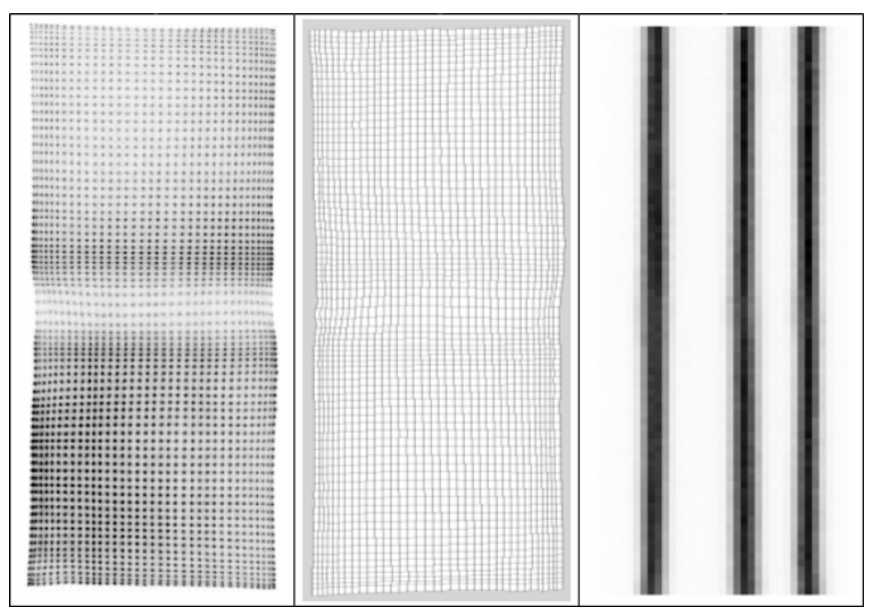

Fig. 2. Raw crystal image (left panel), crystal pixel lookup table (center panel), and an image of three thin capillaries extending from top to bottom (right panel) indicating a negligible loss of sensitivity across the region of low sensitivity between the two PSPMTs.

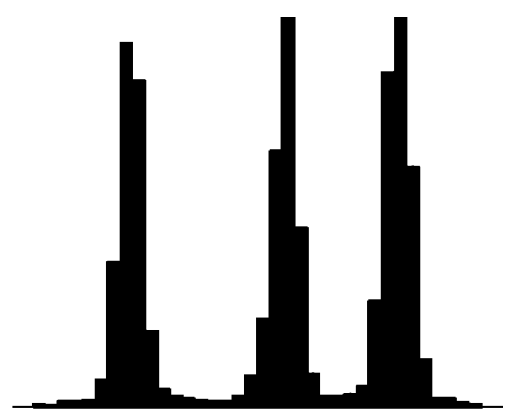

Fig. 3. Profile image (slice, 1 pixel in width) of the three capillaries shown in Fig. 2. The pitch between pixels is $1.2 \mathrm{~mm}(1 \mathrm{~mm}$ pixel size and $0.2 \mathrm{~mm}$ between pixels).

between the two square PSPMTs despite the $\sim 3 \mathrm{~mm}$ insensitive region between photocathode surfaces of the PSPMTs. The "bridging" of the gap that occurs between the two PSPMTs was achieved by light spread affected by the $3 \mathrm{~mm}$ thick optical glass windows of the scintillation array and the $2 \mathrm{~mm}$ thick PSPMT windows and by the use of optical coupling grease between the $\mathrm{NaI}(\mathrm{Tl})$ crystal array window and the entrance window of the PSPMT. The measured energy resolution of the detector for photons from decay of ${ }^{125} \mathrm{I}$ was $35 \%$ FWHM. The energy window was set to accept photons from 22 to $40 \mathrm{keV}$.

Shown in Fig. 4 is a plot of the measured relative efficiency versus distance from a $10 \mu \mathrm{Ci}$ line source of ${ }^{125} \mathrm{I}$ to the face of the collimator. The chief source of uncertainty in the efficiency measurements was the lack of precise source activity and was $\pm 10 \%$. A measure of the sensitivity of this system (including collimator) yields $1840( \pm 10 \%)$ counts/minute $/ \mu \mathrm{Ci}$ as measured with a $6 \mu \mathrm{Ci}$ line source of ${ }^{125} \mathrm{I}(0.3 \mathrm{~mm}$ in diameter $)$ on contact with the outer window. Fig. 5 shows a plot of measured detector spatial resolution (FWHM) versus source-collimator separation obtained from the average of a Gaussian fit to three different one-pixel-wide slices across the line source. One of those slices was in the region of lowered sensitivity between the tubes while the other two were in the top and bottom 


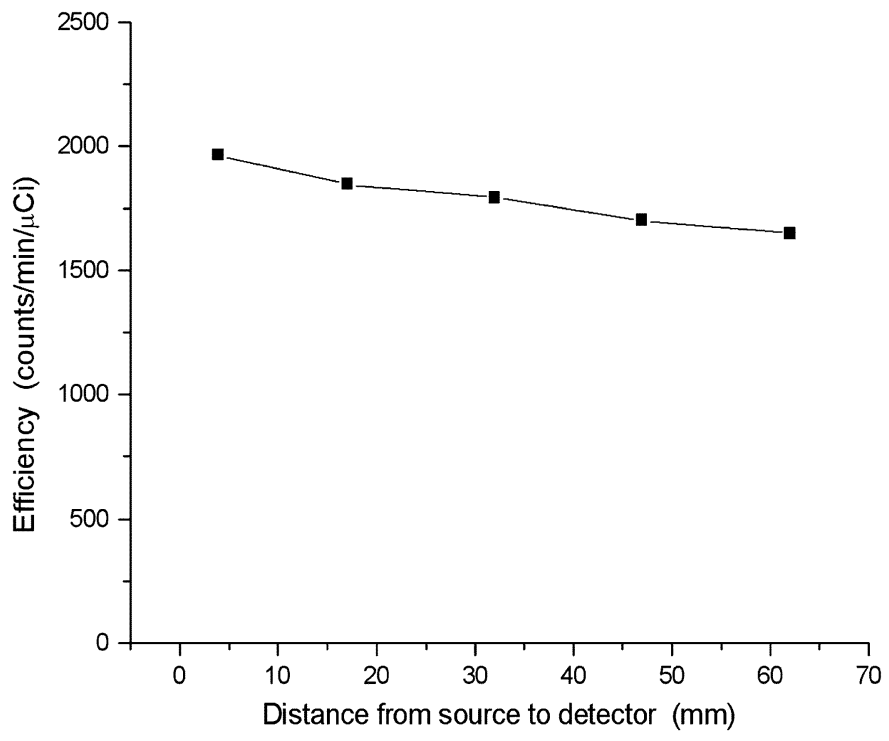

Fig. 4. Detector efficiency (counts $/ \mathrm{min} / \mu \mathrm{Ci}$ ) for a line source of ${ }^{125} \mathrm{I}$ at measured distances $(\mathrm{mm})$ from the collimator face. Absolute values given are limited by precision in determination of the source strength $( \pm 10 \%)$.

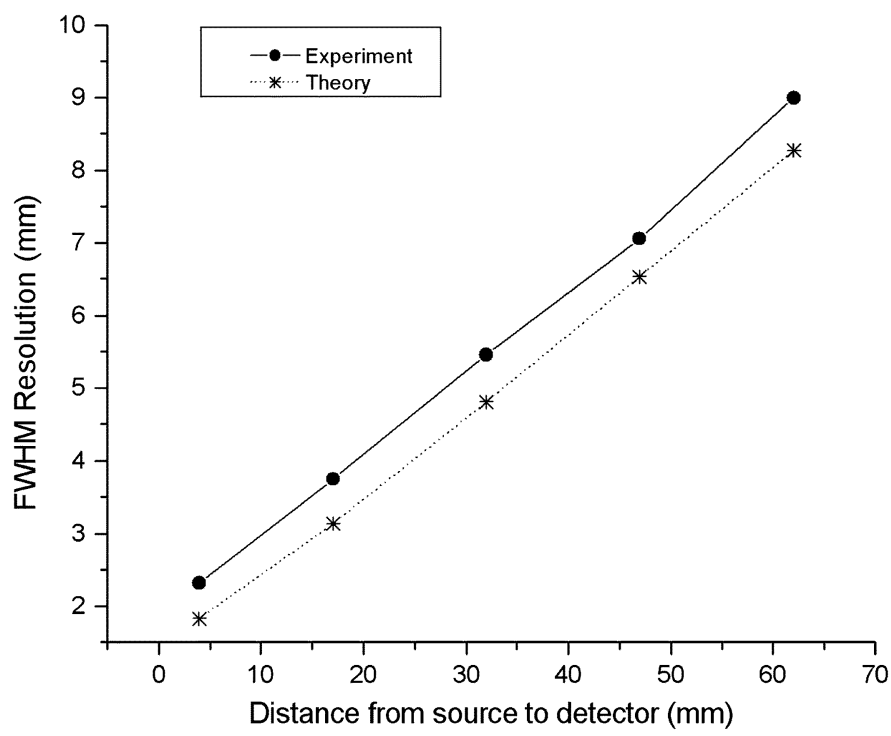

Fig. 5. Resolution (mm) at FWHM for a line source placed at measured heights $(\mathrm{mm})$ above the collimator face. Experimental results are compared with the predictions of [28].

halves of the sensitive area of the detector. Collimator dimensions were based on the formulae of Keller [32] and the more recent work of Smith et al. [28] and the experimental results obtained for resolution and efficiency are in good general agreement with their predictions while noting that the measured resolution curve (Fig. 5) falls about $0.6 \mathrm{~mm}$ FWHM above the theoretical curve. The collimator described in this work has been chosen to provide acceptable system resolution for murine imaging (2.5-3.5 mm FWHM throughout the body of a mouse) with sufficient sensitivity to permit acceptable SPECT reconstruction in a period short enough for imaging of an anesthetized mouse. These collimator parameters also have permitted in-vivo planar imaging at even lower injected activities with the animal in close proximity to the collimator. For higher resolution SPECT imaging, one can employ parallel hole collimation with

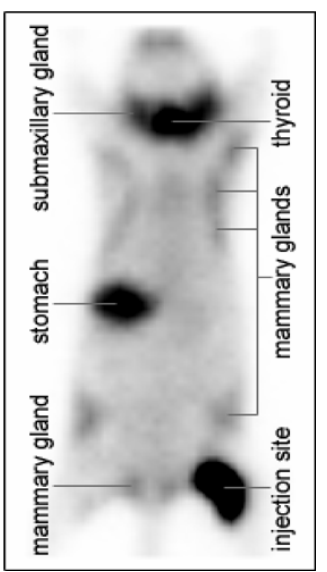

Fig. 6. Image of a 35-g anesthetized female mouse injected with $10 \mu \mathrm{Ci}$ of $\mathrm{Na}$ ${ }^{125} \mathrm{I}$, and positioned on the detector. The injection site was the muscle of the left femur. Evident as well are stomach, thyroid, submaxillary salivary gland region, and each of the mammary glands. The mouth and heart region also show some uptake of the isotope. Each raw pixel is interpolated to yield four smoothed bins in each dimension using NIH Image $\mathrm{J}$.

higher intrinsic resolution and inject correspondingly higher activity into the imaged animal. In addition, as mentioned above, an array of four to six such detectors arranged in close proximity about the animal would allow SPECT studies with a dose as small as $10 \mu \mathrm{Ci}$ of ${ }^{125} \mathrm{I}$.

\section{Results: Animal Imaging Studies}

We have made a preliminary test of the system by imaging a live 35-g lactating female mouse. Normal lactation is known to involve the Sodium (Na) Iodide Symporter (NIS) to transport iodine for the manufacture of milk [33], [34]. The NIS has also been well described in other tissues such as the thyroid salivary glands and stomach. Shown in Fig. 6 is a planar image of the lactating mouse comprised of all the events detected throughout the first 60 minutes following the intramuscular injection of $10 \mu \mathrm{Ci}$ of $\mathrm{Na}^{125} \mathrm{I}$ in $0.10 \mathrm{ml} 0.9 \%$ saline into the left rear leg. In this study, we used intramuscular (IM) injection of the NaI into the femoral biceps, a standard means of injection for many peptide ${ }^{125}$ I ligands, in order to both quantify the injected dose relative to the final distributions as well as to follow the rate and pattern of distribution of the injected dose from a location that was continuously observed. In this image there is demonstrated a clear accumulation of radioactivity in the lactating mammary glands as well as in the stomach positioned asymmetrically on the left side of the animal, and thyroid region, and to a lesser extent in the mouth, heart, and lung region. The injection site in the right rear leg is also apparent. Evident as well is a demarcation between the activity in the thyroid glands and the immediately adjacent submaxillary salivary glands. The resolution of the detector and its applicability to the imaging of ${ }^{125} \mathrm{I}$-containing compounds in mice is clearly demonstrated in this image.

Shown in Figs. 7-9 are images based on a maximum likelihood expectation maximization (mlem) reconstruction [35]. The images represent "slices" spaced $1.2 \mathrm{~mm}$ apart and are based upon reconstruction of 120 planar images of three minutes each taken at $3^{\circ}$ spacing around the animal. Fig. 7 is a transaxial slice of a spect reconstruction in the thyroid and submaxillary 


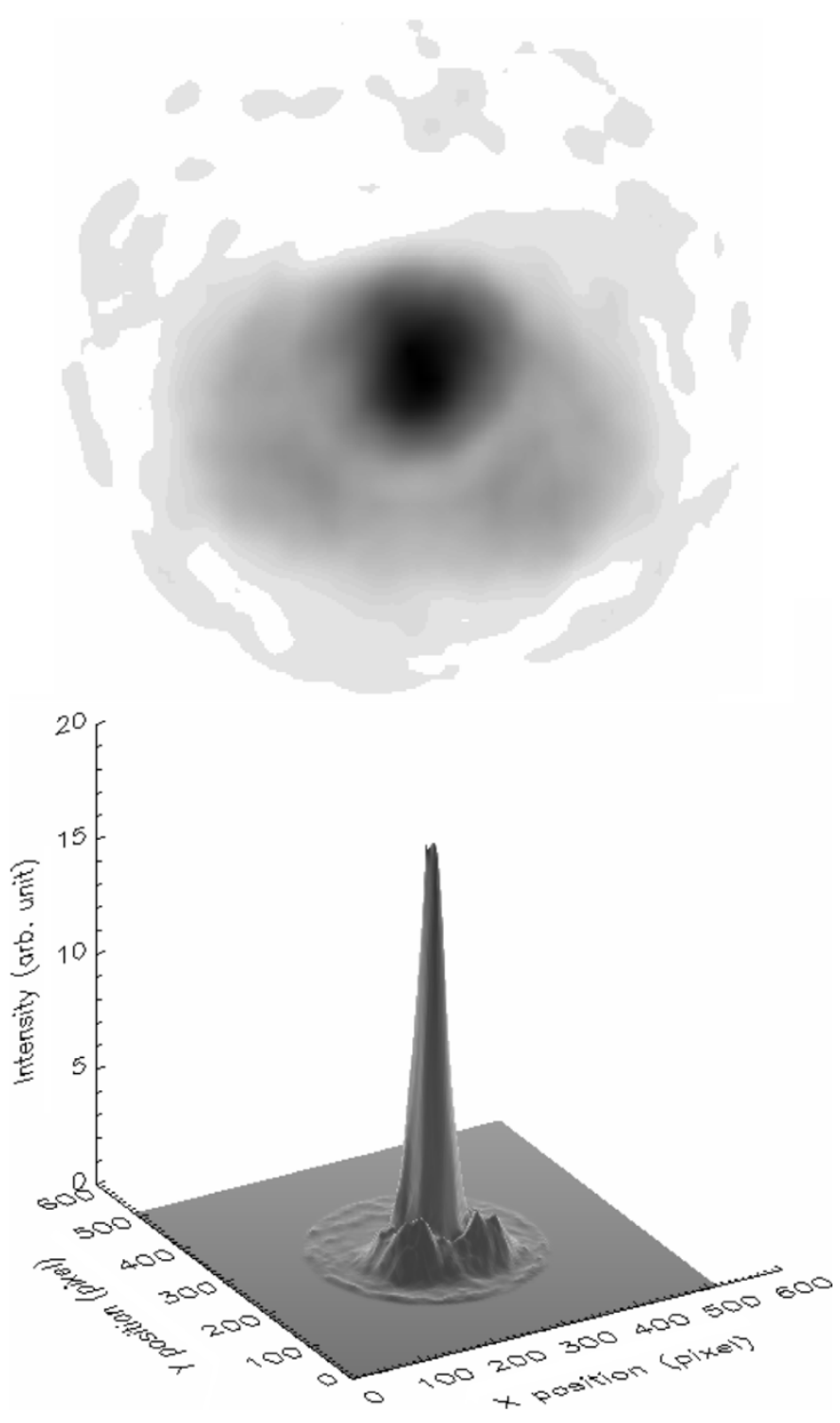

Fig. 7. Top: Transaxial slice of a SPECT reconstruction in the thyroid and submaxillary salivary gland region. The thyroid gland is in the center of the image and the salivary glands are on each side on the ventral (lower) aspect of the image. Bottom: A surface plot of the data from the transaxial slice shown at the top. The center peak represents the intensity in the thyroid gland and the smaller satellite peaks represent the intensity in the two adjacent submaxillary salivary glands on the ventral portion of the slice.

salivary gland region of the mouse shown in Fig. 6, following euthanasia at 65 minutes post injection of the na ${ }^{125} \mathrm{I}$. This slice (Fig. 7, top) indicates the accumulation of ${ }^{125} \mathrm{I}$ in the cross-section that includes the thyroid gland and shows the portions of the two submaxillary salivary glands that are adjacent and ventral to the thyroid. The surface plot of these data (Fig. 7, bottom) illustrates the dramatic difference in the amount of accumulation of ${ }^{125} \mathrm{I}$ in the small area of the thyroid compared with the salivary glands. These data are entirely consistent with the expectation for active iodine accumulation involving the NIS in these two structures.

Figs. 8 and 9 illustrate the distribution of $14 \mu \mathrm{Ci} \mathrm{Na}{ }^{125} \mathrm{I}$ in the body of another euthanized female mouse bearing a mammary tumor. Fig. 8 represents transaxial slices of a SPECT reconstruction of this mouse in the region of the thyroid, submax-

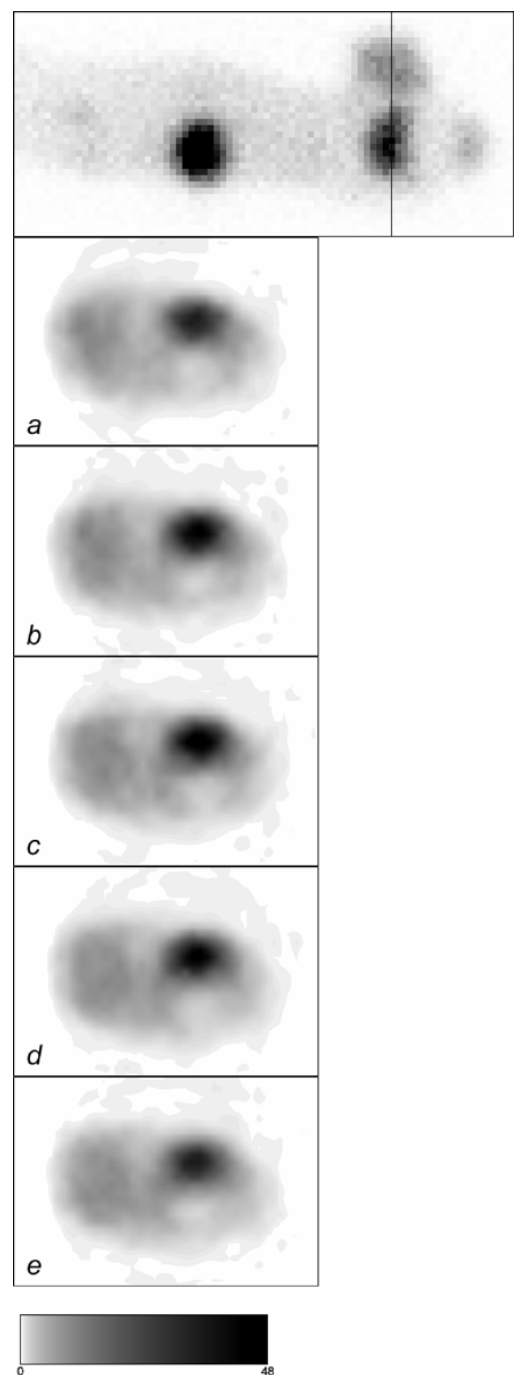

Fig. 8. Top image is a planar view of a mouse bearing a tumor in the right axillary mammary gland. The line locates the position of the centermost slice (c) depicted in the SPECT reconstruction. Transaxial slices (a-e) of a SPECT reconstruction of the mouse are shown. Consecutive slices progress from the caudal end (a) toward the head end (e) in the region of the tumor and the thyroid gland (on the left and right side, respectively). The scale indicates the color assignments (0-48) for the relative activity depicted in all images.

illary glands, and the right axillary mammary tumor. It is evident from these images that the ${ }^{125}$ I present in the tumor volume slices is greater than that contained in the submaxillary glands. The data suggests that the NIS activity in the tumor at this stage of development is substantial, but not as high as in the thyroid gland.

Represented in Fig. 9 are longitudinal slices of the SPECT reconstruction of the same mouse in a plane that includes the stomach, thyroid, submaxillary glands, and the tumor. In this plane of section it is also evident that the tumor has accumulated more ${ }^{125} \mathrm{I}$ in comparison with the salivary glands. In addition, the highly efficient NIS activity expected in the thyroid and the stomach is clearly evident. The fact that the NIS may also be active at various stages of mammary tumor development is expected and offers the potential for further investigations with this animal model using radioiodine to follow the metabolism of both normal and abnormal mammary cells as reflected by 

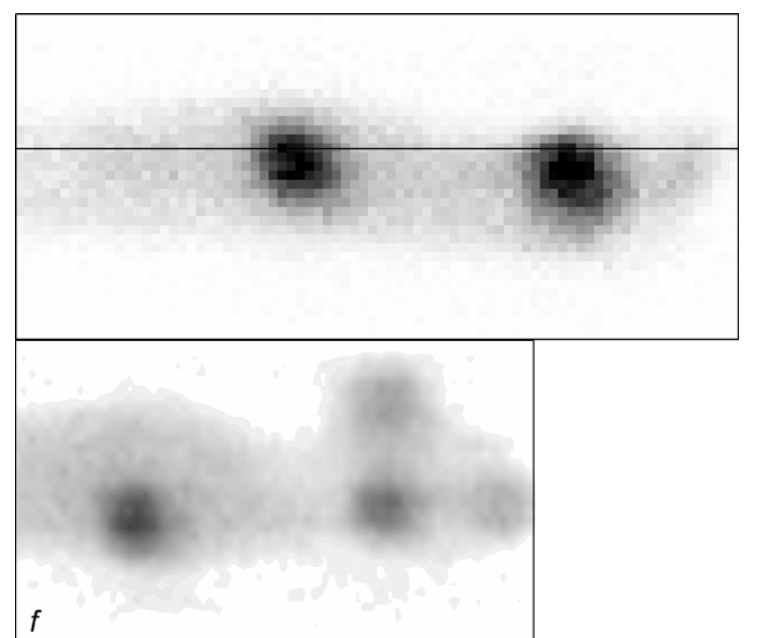

$f$
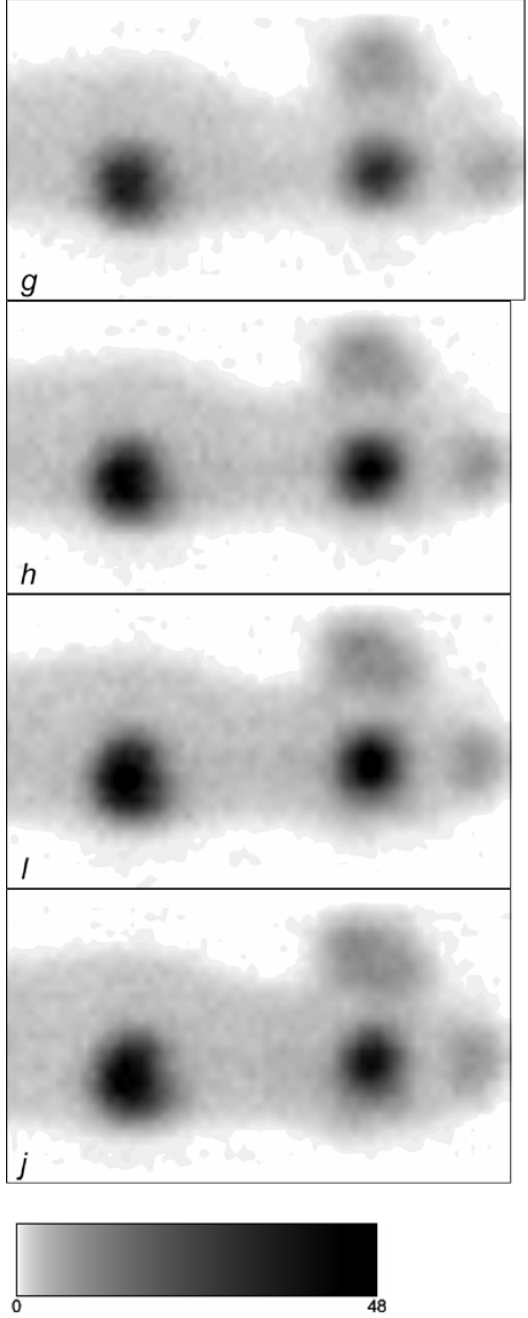

Fig. 9. Top image is a planar view of the mouse bearing a tumor in the right axillary mammary gland. The line locates the position of the center-most slice (h) depicted in the SPECT reconstruction. Coronal slices (f to j) of a SPECT reconstruction of the mouse are shown. Consecutive slices progress from a ventral to a dorsal plane starting just above, and ending just below, a plane containing a major portion of the tumor, thyroid, and stomach. The color scale indicates the color assignments $(0-48)$ for the relative activity depicted in all images.

the expression and relative activity of the NIS in the mammary gland.

\section{CONCLUSION}

We conclude that the detector described here is indeed particularly suitable for imaging mice based on its size, resolution and sensitivity. We note that the combination of two square, flatpanel PSPMTs has been configured to effectively bridge the response gap between the tubes so as to maintain very good uniformity of response (both efficiency and spatial resolution) across the complete detector's active size, and that this detector is able to effectively image an entire mouse. In addition, a compact array of four or five such detectors surrounding a mouse will considerably enhance the ability to carry out SPECT scans with the isotope ${ }^{125} \mathrm{I}$ using physiologically safe levels. By replacing the collimator and increasing the internal radiation shield, the imager can be also applied to imaging higher energy photons such as those from ${ }^{99 \mathrm{~m}} \mathrm{Tc}$.

\section{ACKNOWLEDGMENT}

The authors would like to thank the assistance of B. Kross, K. Smith, and R. Wojcik for valuable contributions to the design of this detector. We are grateful to the William and Mary Physics Machine and Instrument Shop for construction of the case. We appreciate the kind contribution of a sample of Composiflex plastic for use in this detector and thank Dr. S. Meikle of the Department of Radiology at the Royal Prince Albert Hospital in Sydney, Australia, for valuable discussions and for making available to us his IDL computer code for iterative reconstruction.

\section{REFERENCES}

[1] P. D. Acton and H. F. Kung, "Small animal imagining with high resolution single photon emission tomography," Nucl. Med. Biol., vol. 30, pp. 889-895, 2003.

[2] G. Choy, P. Choyke, and S. K. Libutti, "Current advances in molecular imaging: Noninvasive in vivo bioluminescent and fluorescent optical imaging in cancer research," Mol. Imag., vol. 2, pp. 303-312, 2003.

[3] M. Doubrovin, O. Sergampva, P. Mayer-Kuckuk, V. Ponomarev, and R. Blasberg, "Multimodality in vivo molecular-genetic imaging," Bioconjug. Chem., vol. 15, pp. 1376-1388, 2004.

[4] H. R. Herschman, "Noninvasive imaging of reporter gene expression in living subjects," Adv. Cancer Res., vol. 92, pp. 29-80, 2004.

[5] S. Nikolaus, A. Wirrwar, C. Antke, S. Arkian, N. Schramm, H. W. Muller, and R. Larisch, "Quantitation of dopamine transporter blockade by methylphenidate," Eur. J. Nucl. Mol. Imaging, Oct. 12, 2004.

[6] E. E. Graves, R. Weissleder, and V. Ntaizchachristos, "Fluorescence molecular imaging of small animal tumor models," Curr. Mol. Med., vol. 4, pp. 419-430, 2004.

[7] Y. Yang, Y. C. Tai, S. Siegel, D. F. Newport, B. Bai, Q. Li, R. M. Leahy, and S. R. Cherry, "Optimization and performance evaluation of the microPETII scanner for in vivo small-animal imaging," Phys. Med. Biol., vol. 49, pp. 2527-2545, 2004.

[8] E. L. Ritman, "Micro-computed tomography-current status and developments," Аnnu. Rev. Biomed. Eng., vol. 6, pp. 185-208, 2004.

[9] H8500 PSPMT, Hamamatsu, Inc., Hamamatsu City, Japan.

[10] R. Pani, R. Pellegrini, M. N. Cinti, C. Trotta, G. Trotta, F. Garibaldi, R. Scafe, and A. Del Guerra, "Flat panel PMT for photon emission imaging," in Proc. 10th Symp. Radiation Measurements Applications, 2003.

[11] R. Pani, R. Pellegrini, M. N. Cinti, C. Trotta, G. Trotta, R. Scafe, L. D'Addio, G. Iurlaro, L. Montani, P. Bennati, S. Ridolfi, F. Cusanno, and F. Garibaldi, "Factors affecting flat panel PMT calibration for gamma ray imaging," in Proc. 2002 IEEE Nuclear Science Symp. Conf. Rec., 2003.

[12] R. Engels, U. Clemens, G. Kemmerling, and J. Schelten, "High spatial resolution scintillation detector based on the H8500 photomultiplier," in Proc. 2003 IEEE Nuclear Science Symp. Conf. Rec., 2004. 
[13] S. Korpar, I. Adachi, S. Fratina, I. Iijima, R. Islubashi, H. Kawai, P. Krizan, T. Matsumoto, S. Ogawa, R. Pestotnik, S. Saitoh, T. Seki, I. Sumiyoshi, K. Suzuki, T. Tabata, Y. Uchida, and Y. Unno, "Tests of a proximity focusing RICH with aerogel radiator and flat-panel multianode PMT's (H8500)," in Proc. 2003 IEEE Nuclear Science Symp. Conf. Rec., 2004.

[14] C. Lerche, J. M. Benlloch, F. Sanchez, N. Pavon, E. N. Gimenez, M. Gimenez, M. Fernandez, J. Cerda, J. Martinez, and A. Sebastia, "Depth of interaction measurement in gamma ray imaging detectors with continuous scintillation crystals," in Proc. 2003 IEEE Nuclear Science Symp. Conf. Rec., 2004

[15] P. D. Olcott, J. A. Talcott, C. S. Levin, F. Habte, and A. M. K. Foudray, "Compact readout electronics for position sensitive photomultiplier tubes," in Proc. 2003 IEEE Nuclear Science Symp. Conf. Rec., 2004.

[16] V. Popov, S. Majewski, and A. G. Weisenberger, "Readout electronics for multianode photomultiplier tubes with pad matrix anode layout," in Proc. 2003 IEEE Nuclear Science Symp. Conf. Rec., 2004.

[17] A. V. Stolin, M. B. Williams, B. K. Kundu, S. Majewski, V. Popov, and A. G. Weisenberger, "Characterization of imaging gamma detectors for use in small animal SPECT," in Proc. 2003 IEEE Nuclear Science Symp. Conf. Rec., 2004.

[18] N. C. Rouze, M. Schmand, S. Siegel, and G. D. Hutchins, "Design of a small animal PET imaging system with 1 microliter volume resolution," in Proc. 2003 IEEE Nuclear Science Symp. Conf. Rec., 2004.

[19] N. C. Rouze, M. Schmand, S. Siegel, and G. D. Hutchins, "Design of a small animal PET imaging system with 1 microliter volume resolution," IEEE Trans. Nucl. Sci., vol. 51, no. 3, pp. 757-763, Jun. 2004.

[20] Bicron/St. Gobain Inc., Cleveland, OH, Paris, France.

[21] TECOMET, Inc., Woburn, MA.

[22] A. G. Weisenberger, E. Bradley, S. Majewski, and M. Saha, "Development of a novel radiation imaging detector system for in vivo gene imaging in small animal studies," IEEE Trans. Nucl. Sci., vol. 145, no. 3 , pp. 1743-1749, Jun. 1998.

[23] A. G. Weisenberger, E. Bradley, S. Majewski, and M. Saha, "A CsI(Na) based radiation detector for high resolution imaging studies using iodine 125 in small animal research," in Proc. SPIE, vol. 3115, 1997, pp. 254-262.
[24] A. G. Weisenberger, B. Kross, S. Majewski, R. Wojcik, E. Bradley, and M. Saha, "Design features and performance of a CsI(Na) array based gamma camera for small animal gene research," IEEE Trans. Nucl. Sci., vol. 45, no. 6, pp. 3053-3058, Dec. 1998.

[25] R. E. Welsh, E. L. Bradley, B. Kross, S. Majewski, V. Popov, A. Ranck, M. S. Saha, K. Smith, M. F. Smith, A. G. Weisenberger, and R. Wojcik, "An economical dual-modality small animal imaging system with application to studies of diabetes," in Proc. Conf. Rec. IEEE Nuclear Science Symp. Medical Imaging, 2001.

[26] A. G. Weisenberger, R. Wojcik, E. L. Bradley, S. Majewski, J. Qian, A. Ranck, M. S. Saha, K. Smith, M. F. Smith, and R. E. Welsh, "SPECT-CT system for small animal imaging," IEEE Trans. Nucl. Sci., vol. 50, no. 1, pp. 74-79, Feb. 2003.

[27] M. S. Saha, E. L. Bradley, P. Brewer, K. K. Gleason, B. Kross, S. Majewski, V. Popov, J. Qian, A. Ranck, K. Smith, M. F. Smith, A. G. Weisenberger, and R. Wojcik, "Incorporation of a fluoroscopic X-ray modality in a small animal imaging system," IEEE Trans Nucl Sci., vol. 50, no. 3, pp. 333-338, Jun. 2003.

[28] M. F. Smith, S. Majewski, and A. G. Weisenberger, "Optimizing pinhole and parallel hole collimation for scintimammography with compact pixellated detectors," IEEE Trans Nucl Sci., vol. 50, no. 3, pp. 321-326, Jun. 2003.

[29] Composiflex, Inc., Erie, PA, 16509.

[30] RX-688 Optical Coupling Grease. Beachwood, OH, 44 122: Rexon Inc.

[31] Sparrow Corp., Port Orange, FL, 32128.

[32] E. L. Keller, "Optimum dimensions of parallel-hole, multi-aperture collimators for gamma-ray cameras," J. Nucl. Med., vol. 9, pp. 233-235, 1968.

[33] J. Y. Cho, R. Leveille, R. Kao, B. Rousset, A. F. Parlow, W. E. Burak Jr., E. L. Mazzaferri, and S. M. Jhiang, "Hormonal regulation of radioiodide uptake activity and $\mathrm{Na}+/ \mathrm{I}-$ symporter expression in mammary glands," J. Clin. Endocrinol. Metab., vol. 85, pp. 2936-2943, 2000.

[34] B. Perron, A. M. Rodriguez, G. Leblanc, and T. Pourcher, "Cloning of the mouse sodium iodide symporter and its expression in the mammary gland and other tissues," J. Endocrinol., vol. 170, pp. 185-196, 2001.

[35] S. Meikle, private communication personal communication. 